\title{
Association between the RAGE (receptor for advanced glycation end-products) $-374 T$ /A gene polymorphism and diabetic retinopathy in T2DM
}

\author{
Dan $T_{a 0}{ }^{1,2 *}$, Xuancheng Mal ${ }^{3}$, Tiesong Zhang ${ }^{2}$, Yan Mei 33 \\ ${ }^{1}$ Faculty of Environmental Science and Engineering, Kunming University of Science and Technology, Kunming, China \\ ${ }^{2}$ Kunming Children's Hospital, Kunming, China \\ ${ }^{3}$ The First People's Hospital of Yunnan Province, Kunming, China \\ ${ }^{4}$ Faculty of Medicine, Kunming University of Science and Technology, Kunming, China
}

Study conducted by Kunming Health Planning Commission personnel training program (Project number: 2016-sw (backup) -66), Yunnan Science and Technology Planning Project (Kunming Medical Joint Special) (project number: 2017FE468 (-237))

Article received: 4/25/2017 Accepted for publication: 5/7/2017

*Correspondence: Kunming University of Science and Technology Kunming - China Postal code: 650093 taodan1975@163.com

\section{SUMmARY}

Objective: Interaction between advanced glycation end-products (AGEs) and receptor for AGEs (RAGE) in cells could affect both extracellular and intracellular structure and function, which plays a pivotal role in diabetic microvascular complications. The results from previous epidemiological studies on the association between RAGE gene -374T/A polymorphism and diabetic retinopathy (DR) risk were inconsistent. Thus, we conducted this meta-analysis to summarize the possible association between RAGE -374T/A polymorphism and DR risk.

Method: We searched all relevant articles on the association between RAGE -374T/A polymorphism and DR risk from PubMed, Cochrane Library, ScienceDirect, Wanfang, VIP and Chinese National Knowledge Infrastructure (CNKI) web databases up to August 2016. Odds ratio (OR) with 95\% confidence interval (CI) were calculated to assess those associations. All analyses were performed using the Review Manager software.

Results: Nine case-control studies, including 1,705 DR cases and 2,236 controls were enrolled, and the results showed that the A allele of RAGE -374T/A polymorphism was significantly associated with increased DR risk in dominant model (TA/ AA vs. TT: $\mathrm{OR}=1.22$, 95CI 1.05-1.41, $\mathrm{p}=0.006$ ) and heterozygote model (TA vs. TT: $\mathrm{OR}=1.26,95 \mathrm{CI} 1.07-1.47, \mathrm{p}=0.005)$. The subgroup analysis by ethnicity showed that significantly increased DR risk was found in both Asian and Caucasian populations. Conclusion: This meta-analysis reveals that the A allele of RAGE -374T/A polymorphism probably increase DR risk.

Keywords: polymorphism, genetic, diabetic retinopathy, meta-analysis.

\section{INTRODUCTION}

Diabetic retinopathy (DR) is the most common diabetic microvascular complications, affecting approximately $4.8 \%$ of diabetes mellitus patients all over the world. ${ }^{1} \mathrm{DR}$ is one of the most important health threats and the leading cause of blindness in diabetes mellitus patients. ${ }^{2}$ The pathophysiology of DR, characterized as basement membrane thickening, pericyte loss, endothelial cell dysfunction, microaneurysms, microvascular infarcts and neovascularization, is complex and multifactorial, and has not yet been clarified. ${ }^{3-7}$ DR is a complex disease caused by multiple genetic, environmental, and clinical factors. Previous epidemiological studies have identified several risk factors in the pathogenesis of DR, including duration of diabetes, poor disease control, systemic hypertension and proteinuria. ${ }^{8}$ However, these clinical risk factors account only for small portion of the severe forms of DR. Recently, numerous reports have shown that genomics play an important role in the susceptibility and progression of DR. ${ }^{9-11}$

In diabetes mellitus patients, high levels of blood sugar lead to nonenzymatic protein glycation and the formation of advanced glycation end-products (AGE). ${ }^{12}$ AGE could bind to a specific receptor known as receptor for advanced glycation end-products (RAGE). ${ }^{13}$ Many studies have investigated that AGEs and RAGE were over-expressed in DR. ${ }^{14,15}$ The interaction of AGE with 
RAGE could lead to a positive feedback loop that enhances the expression of RAGE in the retina, then activates a number of transcription factors, adhesion molecules and tissue factor, and thus induces oxidative stress, cellular dysfunction, increased vascular permeability, adhesion molecule expression and cytokine production. ${ }^{16-18}$ All of these effects could promote procoagulant and hypoxic state in the microcapillaries of the retina and leads to the initiation of the angiogenic process in proliferative DR. The RAGE gene is located on chromosome $6 \mathrm{p} 21.3$ in MHC Class III region, and the -374T/A polymorphism is the well-characterized genetic variant of this gene. ${ }^{19,20}$ Researches have demonstrated that $-374 \mathrm{~T} /$ A polymorphism of the RAGE gene could result in a Tto-A nucleotide substitution, which leads to prevent the binding of a nuclear binding factor and thus increase RAGE gene transcription activity. ${ }^{20}$ Therefore, RAGE $-374 \mathrm{~T} / \mathrm{A}$ variant may result in susceptibility to and progression of DR.

Numerous case-control studies have observed the association between RAGE -374T/A polymorphism and DR risk. ${ }^{20-28}$ However, their results revealed tremendous disparity that may be attributed to possible selection bias or individual small-sized studies. Thus, we carried out the meta-analysis to distinguish the association between the RAGE -374T/A polymorphism and DR risk.

\section{Method}

\section{Literature search}

Eligible studies were identified using PubMed, Cochrane Library, ScienceDirect, Wanfang, VIP and CNKI web databases with following keywords: "diabetic retinopathy," "RAGE or AGER Receptor for advanced glycation endproducts," "-374T/A or -374A" and "polymorphism or mutation or variant" updated up to October17, 2015. Reference lists of relevant articles were reviewed manually to look for additional studies. We did not restrict language of the article in this process.

\section{Inclusion and exclusion criteria}

Articles included in this meta-analysis were required to meet the following requisites: a) observe an association between -374T/A polymorphisms in RAGE gene and DR risk; b) apply a case-control or cohort study design; c) have enough genotype frequencies in both case and control groups. The major exclusion criteria were: a) duplicated articles in different databases; b) reviews, comments and editorials; c) low-quality studies, for example caseonly studies; d) insufficient data to calculate the effects; e) case groups enrolling type 1 diabetes mellitus (T1DM) patients only. We only included studies presenting good design quality and large sample sizes, in addition to similar patient populations.

\section{Data extraction}

Two reviewers independently extracted the following data from the enrolled studies: The data of the enrolled studies, including authors, country, ethnicity, publication year, source of control, sample size, age, gender frequencies, diabetes mellitus (DM) duration, body mass index (BMI) and hemoglobin A1c (HbA1c) levels, and genotype frequencies in both case and control groups. Disagreements regarding the extracted data between the two reviewers were thoroughly discussed in order to reach a final consensus.

\section{Statistical analysis}

The $\chi^{2}$ analysis was used to test Hardy-Weinberg equilibrium (HWE) in control groups and a p-value $<0.05$ was considered as departure from HWE. ${ }^{29}$ Pooled odds ratio (OR) with 95\% confidence intervals (CI) was calculated to assess the strength of the association between RAGE -374T/A polymorphism and DR risk in allelic comparisons (A vs. T), homozygote (AA vs. TT), heterozygote (TA vs. TT), dominant (AA/TA vs. TT) and recessive models (AA vs. TA/TT), respectively. Heterogeneity among studies was evaluated using $\chi^{2}$ test and $\mathrm{I}^{2}$. If substantial heterogeneity was present $\left(\mathrm{p}<0.10\right.$ or $\left.\mathrm{I}^{2}>50 \%\right)$, the random-effects model was used as the pooling method; otherwise, the fixed-effects model was applied. ${ }^{30,31}$ Finally, publication bias was evaluated using egger's linear regression test. ${ }^{32,33}$ All statistical analyses of this study were conducted by using Review Manager software version 5.2.11 (RevMan; Cochrane Collaboration).

\section{Results}

\section{Characteristics of eligible studies}

The flowchart shows the study selection procedures of this meta-analysis (Figure 1). After careful search and selection, nine case-control studies with 1,705 cases and 2,236 controls were enrolled in this meta-analysis. Table 1 shows the main characteristics of each study. Of the nine studies included, five were performed in Caucasian populations and four in Asian populations.

\section{Association of rs 1057035 and overall cancer susceptibility}

When we pooled all the results of the studies included in our analysis, the results showed that RAGE -374T/A polymorphism was significantly associated with increased DR risk in dominant (TA/AA vs. TT: OR=1.22, 95CI 1.05- 
1.41, $\mathrm{p}=0.006$ ) and heterozygote (TA vs. TT: OR=1.26, 95CI 1.07-1.47, $\mathrm{p}=0.005)$ models. However, there was no association between RAGE -374T/A polymorphism and DR risk in allelic comparison, homozygote model, and recessive model. Subgroup analysis by ethnicity showed that significantly increased DR risk was found in Asian populations (TA/AA vs. TT: $\mathrm{OR}=1.22$, 95CI 1.05-1.41, $\mathrm{p}=0.006 ; \mathrm{AA}$ vs. TT/TA: $\mathrm{OR}=2.92,95 \mathrm{CI}$ 1.47-7.48, $\mathrm{p}=0.030$; AA vs. TT: $\mathrm{OR}=3.17$, 95CI 1.23-8.16, $\mathrm{p}=0.020)$, and in Caucasian populations (AA vs. TT: $\mathrm{OR}=1.24$, 95CI 1.03$1.50, \mathrm{p}=0.030$ ). When stratified by case sample size, significantly increased DR risk was observed in case sample size $\geq 150$ group (TA vs. TT: OR=1.22, 95CI 1.02-1.45, $\mathrm{p}=0.030)$, but not in case sample size $<150$ group in any of the models (Table 2).

\section{Heterogeneity analysis}

There was no significant heterogeneity observed in the allelic comparison (A vs. T), heterozygote model (TA vs. TT), and dominant model (AA/TA vs. TT) for overall analysis $\left(P_{Q}>0.10\right)$, thus, fixed effects model was performed to pool the data. However, significant heterogeneity was observed in the homozygote (AA vs. TT) and recessive (AA vs. TA/TT) models for overall analysis (both $P_{Q}<0.10$ ). Because of that, we performed the subgroup analyses to explore the source of heterogeneity. Although the results indicated that heterogeneity was still significant in Caucasian populations (AA vs. TT/TA: $P_{Q}=0.03$; AA vs. TT: $P_{Q}=0.03$ ) and in case sample size < 150 group (AA vs. TT/TA: $P_{Q}=0.005$; AA vs. TT: $\left.P_{Q}=0.005\right)$, it clearly decreased in Asian populations (AA vs. TT/TA: $P_{Q}=0.88$; AA vs. TT: $P_{Q}=0.87$ ) and in

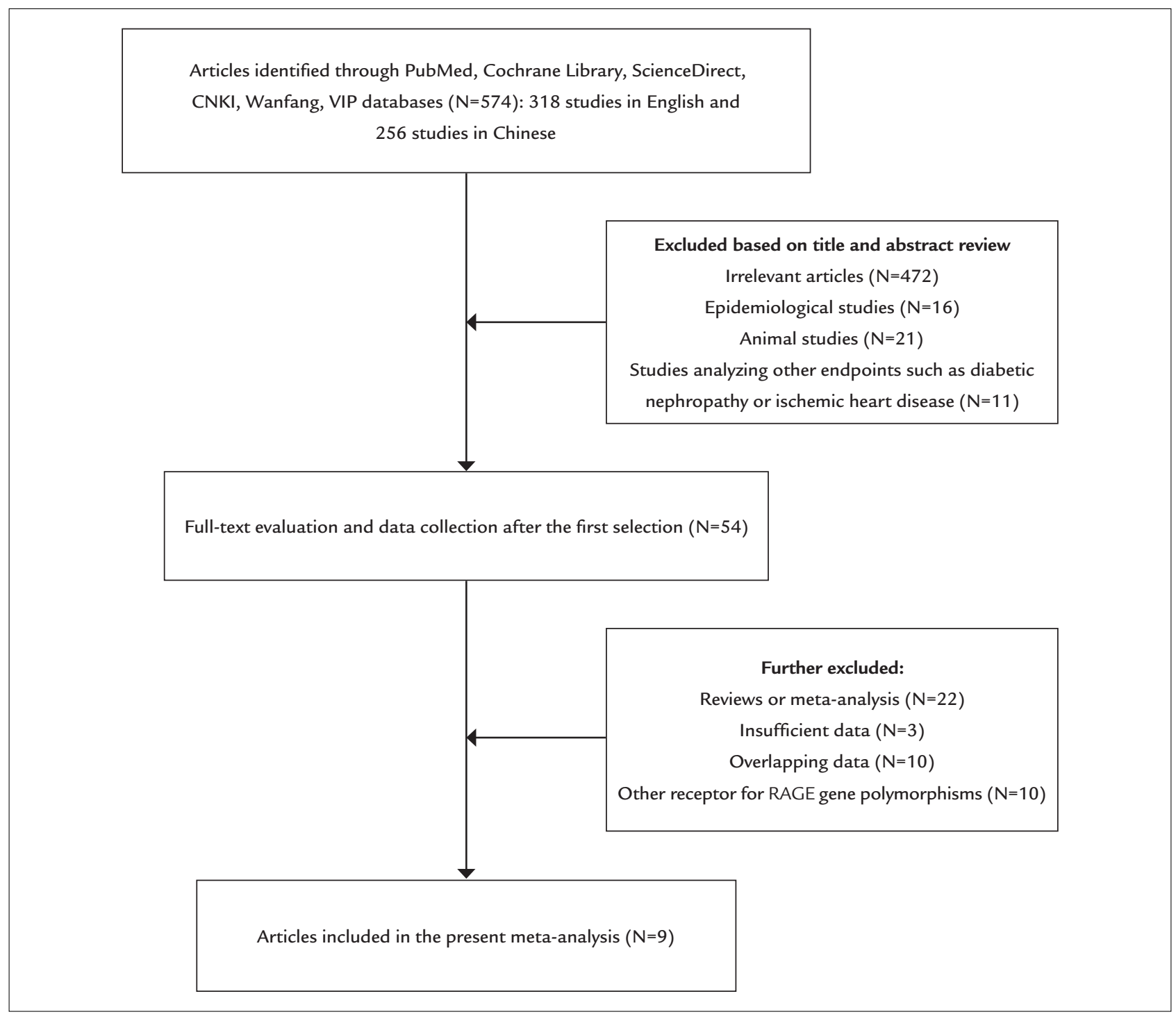

FIGURE 1 Flow diagram of search strategy and study selection. 


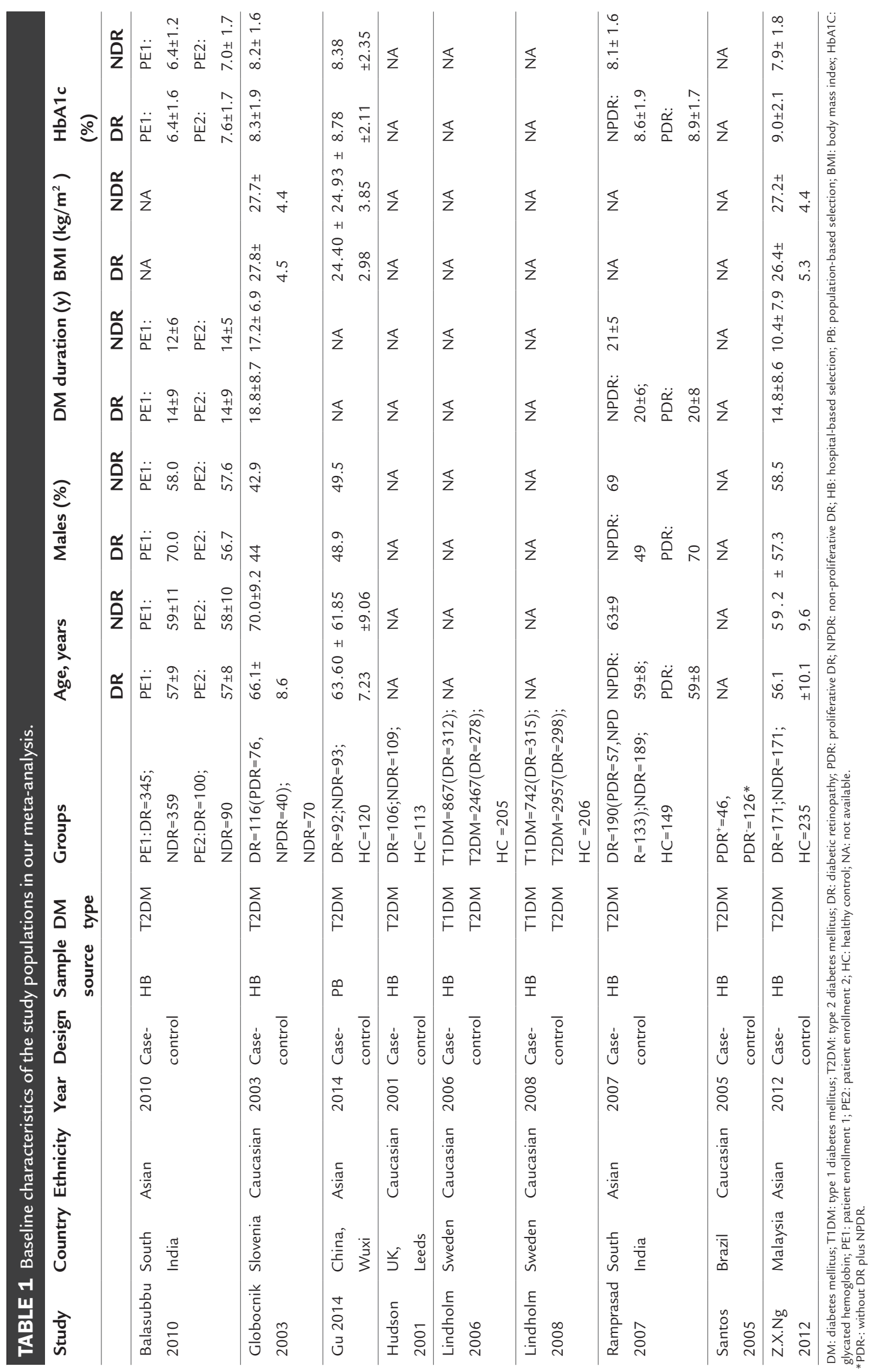




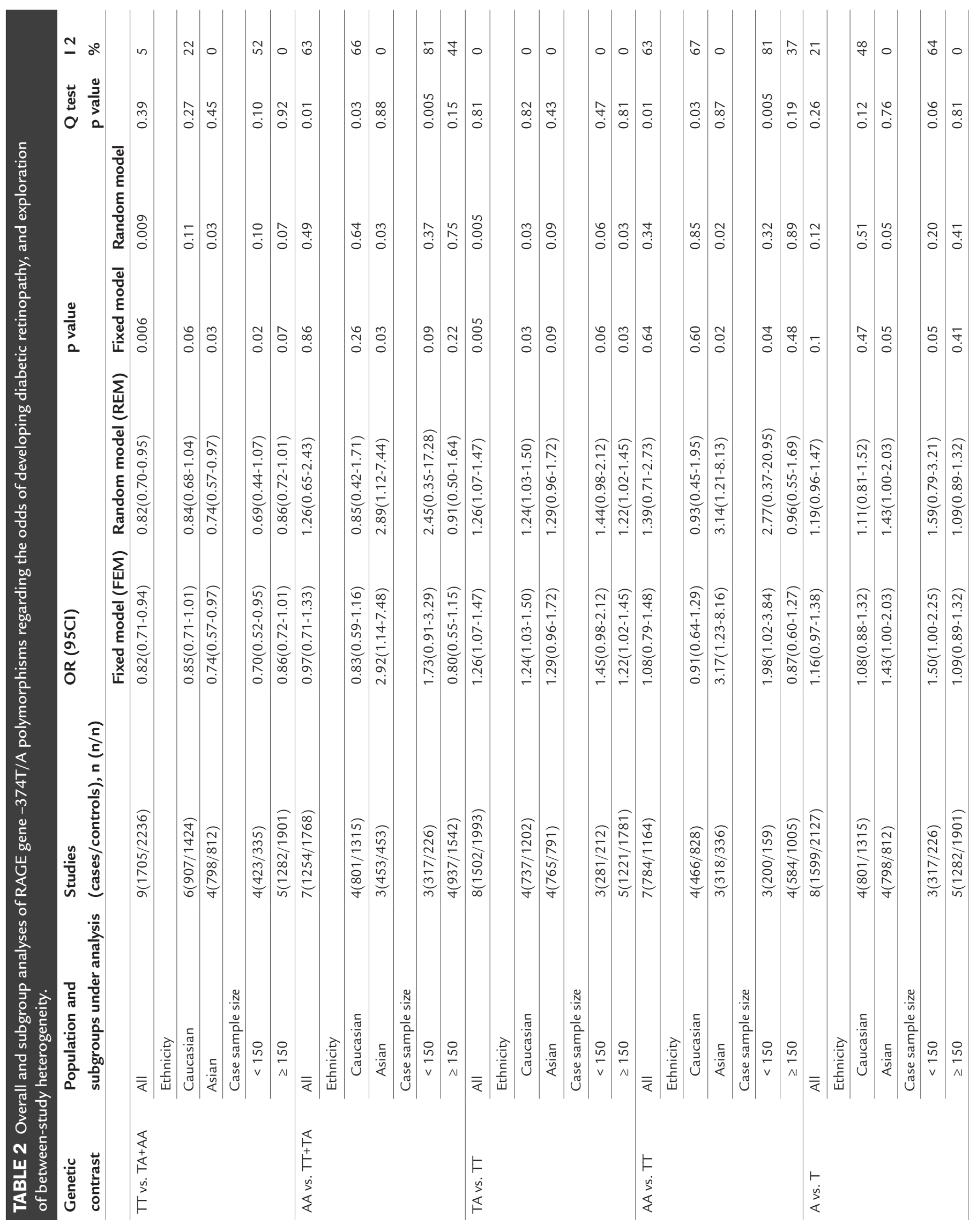


case sample size $\geq 150$ group (AA vs. TT/TA: $P_{Q}=0.15$; AA vs. TT: $\left.P_{Q}=0.19\right)$ (Table 2 ).

\section{Publication bias}

Both the funnel plots and Egger's linear regression test indicated that there was no significant publication bias in any of the above-mentioned inherited models (data not shown).

\section{Discussion}

This meta-analysis included nine case-control studies with 1,705 DR cases and 2,236 controls, and the results showed that the A allele of RAGE -374T/A polymorphism was significantly associated with increased DR risk in the dominant and heterozygote models.

The deregulation of AGEs and RAGE is supposed to play a pivotal role in diabetes mellitus development and progression. ${ }^{34}$ Many studies have investigated that AGEs and RAGE were overexpressed in the target organ of diabetic microvascular complications. ${ }^{35}$ AGEs and RAGE play a complex role in DR through their ability to activate several intracellular cascades, such as NADPH-oxidase/ NF-Kb and p21ras/MAP-kinase/AP-1 pathway. ${ }^{36}$ The interaction of AGE with RAGE could lead to a positive feedback loop that enhances the expression of RAGE in the retina, then induces oxidative stress, cellular dysfunction, increased vascular permeability, adhesion molecule expression, cytokine production and initiation of coagulation, and thus causes a plethora of deleterious effects. ${ }^{16-18}$

Given the critical function of RAGE in oxidative stress and inflammatory response in DR, it is reasonable to suppose that host genomic polymorphism of RAGE may influence DR risk. -374T/A polymorphism is located in the promoter region of the RAGE gene, which could affect RAGE mRNA and protein expression. Recently, several studies have researched the role of $-374 \mathrm{~T} / \mathrm{A}$ polymorphism in the etiology of DR. ${ }^{20-28}$ However, their results were not consistent. One meta-analysis was conducted by Lu et al. ${ }^{37}$ to assess the association of RAGE -374T/A polymorphism with DR risk. However, there were numerous advantages of our research over that of Lu et al. ${ }^{37}$ First, our meta-analysis included a total of nine Chinese populations with 1,705 DR cases and 2,236 controls, which enrolled more studies and subjects than the aforementioned studies. Second, compared with the study by Lu et al., our meta-analysis performed a subgroup analysis that could clarify possible bias from different ethnic populations and sample sizes. ${ }^{37}$ Therefore, our meta-analysis could provide additional precision to characterize the association of the RAGE -374T/A polymorphism with DR risk.
Third, the study by Lu et al. did not find an association of the RAGE -374T/A polymorphism with DR risk in any genetic models. ${ }^{37}$ However, our meta-analysis results showed that the A allele of RAGE -374T/A polymorphism was significantly associated with increased DR risk in both the dominant and heterozygote models. The A allele of RAGE -374T/A polymorphism may prevent the binding of a nuclear binding factor, leading to increased RAGE gene transcription activity, and thus contributes to increase the risk of DR.

In the subgroup analysis by ethnicity, there were different results in Asian and Caucasian populations. One reason may be that the number of studies and number of subjects in Asian and Caucasian populations were relatively small, yielding insufficient statistical power to observe the same association in different populations. The other reason may be that Asian and Caucasian populations have different genetic backgrounds, life-styles, and dietary habits, and therefore produce different degrees of DR susceptibility.

In addition, significant heterogeneity was observed in our meta-analysis. Thus, we performed subgroup analyses stratified by ethnicity and sample size in order to explore the potential sources of heterogeneity, and the results clearly showed less heterogeneity in Asian populations and in case sample size $\geq 150$ group, which was still significant in Caucasian populations and in case sample size $<150$ group. The reason may be that we were not able to perform an analysis adjusted for the demographic characteristics and clinical features, which were also sources of heterogeneity as the unavailable individual data of the eligible studies.

Despite the meaningful results from our meta-analysis, there were also some limitations. First, although we included nine studies with 3,941 subjects to investigate the association of RAGE -374T/A polymorphism with DR risk, sample size was still not big enough to provide adequate statistical power. Second, there are other important genetic polymorphisms involved in AGE-RAGE interaction and the downstream oxidative stress and inflammatory response pathways that may affect the risk of DR, such as AGEs, endothelin 1 , nitric oxide synthase 3 , and lymphotoxin- $\alpha$ gene polymorphisms. Nevertheless, we did not research the potential interactions of the RAGE -374T/A polymorphism with them as there were no sufficient data.

In conclusion, our meta-analysis suggests that the $\mathrm{A}$ allele of RAGE -374T/A polymorphism might confer a moderately augmented risk for DR. We also conducted a subgroup analysis for ethnicity, the potential confounding of DR, to further clarify this association. Further well-designed studies with larger sample sizes and function researches are warranted to validate our findings. 


\section{Conflict of Interest}

The authors declare no conflict of interest.

\section{References}

1. Resnikoff S, Pascolini D, Etya'ale D, Kocur I, Pararajasegaram R, Pokharel $\mathrm{GP}$, et al. Global data on visual impairment in the year 2002. Bull World Health Organ. 2004; 82(11):844-51.

2. Lin S, Ramulu P, Lamoureux EL, Sabanayagam C. Addressing risk factors, screening, and preventative treatment for diabetic retinopathy in developing countries: a review. Clin Exp Ophthalmol. 2016; 44(4):300-20.

3. Ramsey DJ, Arden GB. Hypoxia and dark adaptation in diabetic retinopathy: interactions, consequences, and therapy. Curr Diab Rep. 2015; 15(12):118.

4. Behl T, Kaur I, Kotwani A. Role of endocannabinoids in the progression of diabetic retinopathy. Diabetes Metab Res Rev. 2016; 32(3):251-9.

5. Roy S, Ha J, Trudeau K, Beglova E. Vascular basement membrane thickening in diabetic retinopathy. Curr Eye Res. 2010; 35(12):1045-56

6. Beltramo E, Porta M. Pericyte loss in diabetic retinopathy: mechanisms and consequences. Curr Med Chem. 2013; 20(26):3218-25

7. Behl T, Kotwani A. Exploring the various aspects of the pathological role of vascular endothelial growth factor (VEGF) in diabetic retinopathy. Pharmacol Res. 2015; 99:137-48.

8. Roy MS. Diabetic retinopathy in African Americans with type 1 diabetes: the New Jersey 725: II. Risk factors. Arch Ophthalmol. 2000; 118(1):105-15.

9. Buraczynska M, Zukowski P, Ksiazek K, Wacinski P, Dragan M. The effect of Toll-like receptor 4 gene polymorphism on vascular complications in type 2 diabetes patients. Diabetes Res Clin Pract. 2016; 116:7-13.

10. Kaidonis G, Gillies MC, Abhary S, Liu E, Essex RW, Chang JH, et al. A singlenucleotide polymorphism in the MicroRNA-146a gene is associated with diabetic nephropathy and sight-threatening diabetic retinopathy in Caucasian patients. Acta Diabetol. 2016; 53(4):643-50

11. Zeng Y, Dai F, Yang K, Tang Y, Xu M, Zhou Y. Association between a vascular endothelial growth factor gene polymorphism (rs2146323) and diabetic retinopathy: a meta-analysis. BMC Ophthalmol. 2015; 15:163.

12. Jahan H, Choudhary MI. Glycation, carbonyl stress and AGEs inhibitors: a patent review. Expert Opin Ther Pat. 2015; 25(11):1267-84.

13. Yamagishi S, Fukami K, Matsui T. Crosstalk between advanced glycation end products (AGEs)-receptor RAGE axis and dipeptidyl peptidase-4-incretin system in diabetic vascular complications. Cardiovasc Diabetol. 2015; 14:2.

14. Chen M, Curtis TM, Stitt AW. Advanced glycation end products and diabetic retinopathy. Curr Med Chem. 2013; 20(26):3234-40

15. Zong $\mathrm{H}$, Ward $\mathrm{M}$, Stitt AW. AGEs, RAGE, and diabetic retinopathy. Curr Diab Rep. 2011; 11(4):244-52

16. Glenn JV, Stitt AW. The role of advanced glycation end products in retinal ageing and disease. Biochim Biophys Acta. 2009; 1790(10):1109-16.

17. Yamagishi S, Nakamura K, Imaizumi T. Advanced glycation end products (AGEs) and diabetic vascular complications. Curr Diabetes Ver. 2005; 1(1):93-106

18. Xie J, Méndez JD, Méndez-Valenzuela V, Aguilar-Hernández MM. Cellular signalling of the receptor for advanced glycation end products (RAGE). Cell Signal. 2013; 25(11):2185-97.

19. Sugaya K, Fukagawa T, Matsumoto K, Mita K, Takahashi E, Ando A, et al. Three genes in the human MHC class III region near the junction with the class II: gene for receptor of advanced glycosylation end products, PBX2 homeobox gene and a notch homolog, human counterpart of mouse mammary tumor gene int-3. Genomics. 1994; 23(2):408-19.
20. Hudson BI, Stickland MH, Futers TS, Grant PJ. Effects of novel polymorphisms in the RAGE gene on transcriptional regulation and their association with diabetic retinopathy. Diabetes. 2001; 50(6):1505-11.

21. Balasubbu S, Sundaresan P, Rajendran A, Ramasamy K, Govindarajan G, Perumalsamy $\mathrm{N}$, et al. Association analysis of nine candidate gene polymorphisms in Indian patients with type 2 diabetic retinopathy. BMC Med Genet. 2010; 11:158.

22. Globocnik Petrovic M, Steblovnik K, Peterlin B, Petrovic D. The - 429 T/C and - $374 \mathrm{~T} / \mathrm{A}$ gene polymorphisms of the receptor of advanced glycation end products gene are not risk factors for diabetic retinopathy in Caucasians with type 2 diabetes. Klin Monbl Augenheilkd. 2003; 220(12):873-6.

23. Lindholm E, Bakhtadze E, Sjögren M, Cilio CM, Agardh E, Groop L, et al The $-374 \mathrm{~T} / \mathrm{A}$ polymorphism in the gene encoding RAGE is associated with diabetic nephropathy and retinopathy in type 1 diabetic patients. Diabetologia. 2006; 49(11):2745-55

24. Lindholm E, Bakhtadze E, Cilio C, Agardh E, Groop L, Agardh CD. Association between LTA, TNF and AGER polymorphisms and late diabetic complications. PLoS One. 2008; 3(6):e2546.

25. Ramprasad S, Radha V, Mathias RA, Majumder PP, Rao MR, Rema M. Rage gene promoter polymorphisms and diabetic retinopathy in a clinic-based population from South India. Eye (Lond). 2007; 21(3):395-401.

26. Santos KG, Canani LH, Gross JL, Tschiedel B, Pires Souto KE, Roisenberg I. The -374A allele of the receptor for advanced glycation end products gene is associated with a decreased risk of ischemic heart disease in African-Brazilians with type 2 diabetes. Mol Genet Metab. 2005; 85(2):149-56.

27. Ng ZX, Kuppusamy UR, Tajunisah I, Fong KC, Chua KH. Association analysis of $-429 \mathrm{~T} / \mathrm{C}$ and $-374 \mathrm{~T} / \mathrm{A}$ polymorphisms of receptor of advanced glycation end products (RAGE) gene in Malaysian with type 2 diabetic retinopathy. Diabetes Res Clin Pract. 2012; 95(3):372-7

28. Gu ZY, Yao Y, Xie TH, Cao J, Zou J. Association analysis of advanced glycation end products gene receptor -374T/A polymorphisms in Han people of Wuxi area with type 2 diabetic retinopathy. Rec Adv Opthalmol. 2014; 34(7):655-7.

29. Schaid DJ, Jacobsen SJ. Biased tests of association: comparisons of allele frequencies when departing from Hardy-Weinberg proportions. Am J Epidemiol. 1999; 149(8):706-11

30. Zintzaras E, Ioannidis JP. HEGESMA: genome search meta-analysis and heterogeneity testing. Bioinformatics. 2005; 21(18):3672-3.

31. DerSimonian R, Laird N. Meta-analysis in clinical trials. Control Clin Trials 1986; 7(3):177-88.

32. Begg CB, Mazumdar M. Operating characteristics of a rank correlation test for publication bias. Biometrics. 1994; 50(4):1088-101.

33. Egger M, Davey Smith G, Schneider M, Minder C. Bias in meta-analysis detected by a simple, graphical test. BMJ. 1997; 315(7109):629-34.

34. Ramasamy R, Yan SF, Schmidt AM. Receptor for AGE (RAGE): signaling mechanisms in the pathogenesis of diabetes and its complications. Ann N Y Acad Sci. 2011; 1243:88-102.

35. Soro-Paavonen A, Forbes JM. Novel therapeutics for diabetic micro- and macrovascular complications. Curr Med Chem. 2006; 13(15):1777-88.

36. Lander HM, Tauras JM, Ogiste JS, Hori O, Moss RA, Schmidt AM. Activation of the receptor for advanced glycation end products triggers a p21(ras) dependent mitogen-activated protein kinase pathway regulated by oxidant stress. J Biol Chem. 1997; 272(28):17810-4

37. Lu W, Feng B. The -374A allele of the RAGE gene as a potential protective factor for vascular complications in type 2 diabetes: a meta-analysis. Tohoku J Exp Med. 2010; 220(4):291-7. 